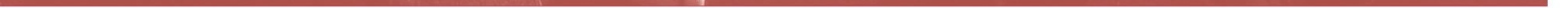




\section{Presentación: Lo que tiene que cambiar para que (casi) todo cambie}

\section{Jorge Fernández León}

\section{Introducción}

En esta segunda parte del dossier se reúne una suma de miradas plurales que no se limita a explorar la memoria del pasado, sino que trata de contribuir a un ejercicio prospectivo ante las transformaciones profundas que la vida cultural de la ciudadanía viene experimentando en las últimas décadas. Los rastros de algunas de las consecuencias del salto digital, con sus valores y conflictos consiguientes, son ya evidentes en el comportamiento cultural de la ciudadanía.

Las certezas cargadas de optimismo del tiempo del crecimiento ilimitado que soñaba el mundo moderno hace tiempo que han llegado a su fin; en eso coinciden críticos culturales tan distantes en su apreciación general como D. Bell (1977), M. Berman (1982) o T. Eagleton (2017).

Uno de nuestros críticos más visionarios, Juan Cueto ${ }^{1}$, describía así el panorama a principios de los ochenta:

Si durante la era de la segunda industrialización fue necesario utilizar la euforia para integrar al ciudadano en el universo del derroche, del consumo, ahora es necesario manipular el pesimismo para lograr una nueva forma de integración. Esas lógicas del sobresalto, de la liquidación de los modelos de la estabilidad y del optimismo, del miedo como medio de comu- nicación de masas, anulan la pluralidad, suspenden la normalidad, recuperan las antiguas disidencias y heterodoxias políticas y sindicales y canonizan lo establecido. Pero al mismo tiempo originan nuevas formas extremas de lucha social, violentas en ocasiones, desesperadas, imprevisibles siempre [...] la incertidumbre produce más incertidumbre

La formación de los mecanismos de intermediación cultural desde finales de los años setenta en España y la constitución de nodos y redes entre los escasos proyectos culturales públicos existentes hace cuatro décadas era solo el deseo aislado de algunos gestores con experiencia internacional. Estaba aún lejos el tiempo para que las articulaciones de las transformaciones culturales pudieran encontrar paralelo alguno a las que, por ejemplo, despliega en sus mapas del presente A. Baricco (2019: 259-273) para intentar explicar los pasos de las transformaciones de la cultura en nuestro universo digital; o el momento en el que los comportamientos de la comunidad consumidora de entonces fueran reconocibles en nuestros municipios de la manera en que los describe P. Slotedijk (2002: 17), cuando habla de la esencia manipulable de la masa contemporánea: «Individuos que en su `desamparo organizado' forman la materia prima de todo experimento 
pasado y futuro de dominio totalitario y mediático». Identificar, además, el marco general que determina los mecanismos de consumo en el capitalismo de vigilancia actual ${ }^{2}$, llevarlo al ámbito local e imaginar siquiera cómo puede actuarse desde lo cercano para responder eficazmente a un reto de esta magnitud será un desafío de dimensiones casi inimaginables hoy. En palabras del profesor Lyons ${ }^{3}$ (2018):

El capitalismo de vigilancia y la cultura de vigilancia no pueden ser estudiadas desapasionadamente, por mucho cuidado y precisión con que sean presentados los datos sociales. Afectan a las identidades, como la gente se ve y se presenta (subjetividades) oportunidades vitales, las formas en que se abren y cierran las oportunidades de acceso dependiendo del consumidor y otras categorías en las que hoy todos estamos ubicados (clasificación social, socioestratificación) y en la participación democrática o hasta donde podemos votar o en qué medida ese voto nos identifica (política). En pocas palabras, la vida humana está afectada negativamente -dicho brevemente en relación a sus subjetividades, su clasificación social y su capacidad combativa- por el capitalismo de vigilancia

Este nuevo escenario, que ha penetrado ya en nuestros barrios y modula cada día la vida de la ciudadanía local, reclama nuevas formas de intervención crítica en la vida cultural de las comunidades. Entre entonces y ahora, las transformaciones sucedidas hacen que, para hacer buen uso de la experiencia de gestión cultural local, debamos recurrir a una cada vez mayor pluralidad de visiones, categorías y experiencias. Ya no es suficiente contar con las viejas herramientas si no queremos que en el muy próximo futuro los valores de la cultura se identifiquen exclusivamente con los del consumo manipulado de los mercados del entretenimiento y acabemos engullidos por los Índices de Felicidad ${ }^{4}$ y otros mecanismos similares que nos asuelan. El territorio, la sostenibilidad, el desarrollo, los conflictos culturales, territoriales y sociales, los derechos de acceso al disfrute y a la producción, los valores de la diferencia, las formas en que se organiza la convivencia... penetran los textos que a continuación se incluyen y tratan de contribuir un poco a incorporar nuevos fragmentos que ayuden a descifrar el complejo mapa de la vida cultural local, tan necesitado de compromisos y colaboraciones como de normas y garantías para su desarrollo democrático.

\section{El panorama español}

Los aniversarios se anuncian casi siempre con aroma de nostalgia inminente. $\mathrm{O}$ de melancolía. Cuando hace un año, ante la inevitable llegada del cuarto decenio, los responsables de Periférica aceptaron la propuesta de incluir en este número un apartado destinado a recordar los avatares de las políticas culturales locales en España, lo hacían sabiendo de otras iniciativas que a lo largo de 2019 conmemorarían el hecho ${ }^{5}$ con análisis generales y sectoriales del mismo.

Así que, para evitar repeticiones, se ha tratado de solicitar a quienes, generosamente, colaboran con sus textos, que el conjunto de fragmentos que componen el dossier abordara algunos de los asuntos cualitativos de la agenda cultural del país, en un momento en el que, como señalaban en un artículo Rubio y Ríus ${ }^{6}$ entre las tareas futuras de las ciencias sociales, se ciernen serias dudas sobre el futuro de la esfera pública de la cultura ${ }^{7}$, que requieren respuestas estructurales y una previa reflexión multilateral:

Así, propondremos lo que según nuestro punto de vista $[\ldots]$ constituyen las futuras tareas de las ciencias sociales en relación a la política y la gestión cultural para buscar una salida alternativa al actual estado de crisis y disolución de la esfera cultural: la búsqueda de nuevos objetivos y legitimaciones de la política cultural, la crítica en la actual gobernanza de la cultura y la transformación en la forma de gestionarla y, la necesidad de buscar nuevas regulaciones y de replantear la participación cultural inscribiéndola en los procesos de innovación social en curso en la entorno digital y de los movimientos sociales

Aunque no esté siendo España un país exigente a la hora de analizar y contextualizar su pasado reciente, el número de estudios históricos y de aproximaciones personales a los procesos culturales dispone de una bibliografía de calidad en muchas de las áreas de la creación. Recordemos algunos de ellos: los trabajos de Marzo y Mayayo (2015) y de Ramírez (ed.) (2010) abordan con brillantez y de forma exhaustiva tanto el sistema del arte en España como el marco más general de las políticas del mismo. Florenci Guntín (1998) contempla otros aspectos más vinculados a la política de las artes en el ámbito local. La historia menos contada de la creación cultural alternativa está recogida con brillantez, aunque de forma incompleta por Costa (2018), así como por distintas publicaciones memorialistas de protagonistas de la contracultura española como García Pelayo (2003), Franco 
Ya no es suficiente contar

\section{con las viejas herramientas}

\section{si no queremos que en el}

\section{muy próximo futuro}

los valores de la cultura

se identifiquen

exclusivamente con los

del consumo manipulado

de los mercados del

entretenimiento... de la Cultura y el Desarrollo (OIKOS $)^{12}$, Debats ${ }^{13}$ o Arte, Individuo y Sociedad ${ }^{14}$ entre otros muchos esfuerzos editoriales, han publicado ensayos, estudios y análisis de casos de buenas y mejores prácticas locales. Varios escritores, en fin, encabezados por Guillem Martínez, desarrollan incluso una muy tendenciosa aunque sugerente descripción de las políticas culturales españolas desde la transición, proponiendo el impreciso concepto de CT o Cultura de la Transición ${ }^{15}$ para explicar su visión del asunto.

Pero la historia de la explosión, crecimiento y conflictos de edad de las políticas culturales locales, desde sus aciertos y limitaciones hasta al detalle de las estrecheces de un largo período de resiliencia iniciado hace una década y sin aparente solución a la vista, sigue sin ser contada en su complejidad y diferencia. Algunos analistas, por experiencia propia, señalaban ya hace tiempo las condiciones generales inaceptables en las que se desarrollaban en el terreno local las políticas culturales de proximidad. Ya hace casi dos décadas, en una publicación ${ }^{16}$ de la Federación Española de $\mathrm{Mu}$ nicipios y Provincias (FEMP), Jordi Font, escribía que: «La política cultural arrastra un extraordinario déficit de objetivación como ninguna otra política de gobierno. Es el marco de la relación entre poder y cultura, una relación cargada de inercias y connotaciones, que ha dado lugar a múltiples derivas». Y, a la hora de enjuiciar los primeros veinte años de acción cultural local, señalaba (pág. 22) que

mientras que la Administración Local provee a los ciudadanos la mayor parte de los servicios culturales de que disponen, su financiación, en términos estructurales globales o mediante concertación sectorial específica con las otras administraciones, sigue absolutamente estancada. Además, los servicios culturales ni siquiera tienen la consideración de factor de modulación del gasto por habitante asignado en las fórmulas generales de la financiación local. Se produce así una aplicación perversa, escandalosa, del principio de subsidiariedad

Poco que añadir a este diagnóstico. Diez años más tarde, se publicaba el monográfico Treinta años de políticas culturales en España (RUBIO ARÓSTEGUI Y RIUS-ULLDEMOLINS, 2016), un amplio intento panorámico de resumir los factores determinantes de tres décadas democráticas en la materia a través del análisis del sistema cultural, sus agentes, las industrias, la participación y el marco regulador y polí-

tico. Pero en este trabajo tampoco, por sus características,
(2004), Malvido (2004), Ordovás (2017), Vogel (2017) y, sobre todo, en las desinhibidas memorias de Nazario (2017, 2018). Entre los citados, dos de ellos, Ordovás y Vogel, hacen historia de la cultura escrita (literatura, pensamiento) tiene en los trabajos de Jordi Gracia $(2006,2009)$ una excelente aproximación desprejuiciada.

proximidad, con investigadores culturales de larga tradición como Juan Arturo Rubio Aróstegui ${ }^{8}$ (2003) - otro de los autores que nos acompaña en el monográfico - solo o en colaboración con Joaquín Ríus Ulldemolins ${ }^{9}$ (2015, 2016) Mencionaremos, además, sin afán exhaustivo, a varios autores con numerosas publicaciones sobre la materia, como Arturo Rodríguez Morató ${ }^{10}$ o Pau Rausell ${ }^{11}$. Todos ellos y una larga lista de investigadores publican, en libros y revistas profesionales, diversos análisis globales sobre la acción cultural de los Gobiernos nacionales, autonómicos y algunos de los locales. Zafra (2017) examina con extraordinaria finura las nuevas formas de la precariedad en el trabajo creativo en este tiempo digital. Publicaciones como Periférica, que se ha demostrado capaz de llegar a la veintena, o la más efimera revista del Observatorio Andaluz para la Economía 
cabía un espacio significativo destinado a la reflexión sobre lo local.

Cuando hace cuatro décadas leíamos la historia de una experiencia popular como la de la Universidad Popular de Rekaldeberri ${ }^{17}$ comenzábamos muchos a descubrir que la acción cultural tenía en el barrio, en la proximidad, también en España, su lugar natural. Ya entonces algunos adelantados, desde Joaquim Jordá ${ }^{18}$ a Eduard Delgado ${ }^{19}$ venían situando el lugar de la cultura como un espacio dinámico de conflictos en el que convivían formas de producción artística y creativa individuales y colectivas con crecientes potentes industrias culturales y del entretenimiento ${ }^{20}$. Una de las presencias constantes en esta tarea ha sido la de la Diputación de Barcelona, pionera en el desarrollo de estrategias y redes culturales locales $^{21}$. Uno de los colaboradores de este monográfico, quien ha sido durante décadas su responsable técnico político, Jordi Font, es testigo excepcional de esa singularidad. Desde su larga experiencia institucional, ahora como secretario del Consell Nacional de la Cultura i de les Arts de Catalunya (CONCA), nos da algunas de las claves de lo que, a su entender, ha de cambiar en las políticas culturales para que su presencia en los programas y en la acción de las instituciones locales siga teniendo sentido.

\section{Una crisis europea: ascensión y caída de las políticas culturales}

La conservación y desarrollo del capital cultural como valor de uso de las comunidades fue hace medio siglo un factor central de las políticas de cambio - especialmente en Europa - y un camino eficaz para la universalización del acceso a un conjunto de derechos universales en distintos países: lectura pública, educación cultural, museos, patrimonio y memoria histórica, lenguas locales, artes en vivo o atención a la diversidad fueron incorporándose, mejor o peor, a la agenda política de los países en nuestro continente y en muchas naciones de América.

Ese discurso era asumido por los partidos socialdemócratas y por las organizaciones progresistas, teórica o prácticamente, en sus discursos o en su acción de gobierno. Pero la penetración de las ideas neoliberales del mérito y el esfuerzo como conductores de la creación frente a los valores de comunidad y compromiso igualitario, junto con la insistencia ciega de muchos en ponderar la acción cultural en relación con su retorno económico mensurable, han ido convirtiendo lo que era valor de uso en mercadería de cambio. Hoy mismo, hasta muchos de quienes trabajan en el sector público, van aceptando más la cultura como mercancía que como factor transformador. Asistimos pues a la mutación del capital cultural $^{22}$ en mero capitalismo cultural, en el que los productos se asimilan de inmediato a mercancías de consumo y se miden por su precio.

Ahora resulta que, entre idas y vueltas, parece que algunos países anglosajones, antes que Francia o Alemania, han ido descubriendo últimamente que se han pasado alrededor de dos décadas sin política cultural. $\mathrm{O}$ al menos eso dicen algunos reputados analistas en materia de economía de la cultura, y arquitectos en la sombra de esta mutación mayor en el panorama europeo e internacional, como es el caso de NESTA, el influyente centro de reflexión creado bajo los auspicios de la Tercera Vía de Tony Blair. Hace tres años, en su documento titulado "Política Cultural en el tiempo de las Industrias Creativas" ${ }^{\text {"23 }}$, que ellos mismos catalogan como «provocación», dos de sus analistas principales lo sostienen sin ningún género de dudas. Lo mismo que hace el informe de la Comisión Warwick ${ }^{24}$, un grupo de trabajo de academia y sociedad que reúne a un buen número de especialistas en estudios culturales, instando a la recuperación de unas políticas culturales sepultadas por la novedad y por los intereses de la economía del entretenimiento.

Todos ellos están aceptando la precisa autopsia que desde hace tres décadas venían realizando algunos críticos culturales [HEWISON (1987), HORNE (1984, 1986)] y hace menos de una certificaban otros $^{25}$, ya con datos: que las políticas culturales, una vez asociadas a las llamadas industrias creativas, han perdido del todo su sentido. Dos décadas después de que, primero en Australia y luego en el Reino Unido $^{26}$, como una novedad del discurso de la socialdemocracia más mediática, se acuñaran los términos Industria Creativa, Economía Creativa y sus derivados ${ }^{27}$. Ello sirvió para volcar los esfuerzos y recursos de los gobiernos de toda Europa, incluida la propia Unión Europea ${ }^{28}$, en el desarrollo prioritario de un sector económico que aprovechara las ventanas de oportunidad del tiempo libre universalizado, frente a cualquier política estructural de garantías de derechos.

\section{4. ¿Décadas casi perdidas?}

Estos estudios citados alertan sobre el peso de veinte años medio perdidos allí — pero también aquí- para las políticas culturales. Algo que entonces, a finales del pasado milenio, ya percibíamos en España, cuando los gobiernos, nacional y autonómicos, ralentizaron el proceso de transferencia de recursos a los entes locales para sus políticas de proximidad. Los municipios, asumiendo cada vez más responsabilidades de gestión sin financiación adecuada, comen- 
zaron a reducir el flujo de innovación y transformaciones y la disrupción dejó paso a la costumbre. Ello nos ha ido llevando a un deterioro progresivo, continuado, de la innovación de los servicios culturales locales, humus de la creación vital para un país. Y ese empobrecimiento ha ido creciendo, de la raíz a la copa, dejando rastros en la escuálida presencia de la creación en la constitución de la identidad simbólica de regiones y del país entero. Sin políticas de territorio que transciendan de las operaciones cortoplacistas de prestigio y con una ausencia flagrante desde hace casi una década de cualquier estrategia nacional creíble por parte del Gobierno de España ${ }^{29}$.

\section{Cosas que hacer para que mucho (o algo)}

\section{cambie}

Este escenario, cuarenta años después de las primeras elecciones democráticas tras el franquismo, no puede resolverse, no obstante, con descalificaciones o simplificaciones como la CT, olvidando el trabajo de comunidades enteras que han demostrado que sí es posible una práctica cultural democrática, cercana y compartida. Son muchos los escenarios en los que hay que pensar y actuar, si se quiere devolver a las políticas culturales el papel articulador de la vida comunitaria que les corresponde. Políticas para devolver a la cultura su valor de uso y su constitución como un derecho de iguales. Escenarios que ahora necesitan, más que nunca, de algo más que una profunda revisión crítica. $\mathrm{O}$, por el contrario, de preparativos para unos fastuosos funerales. La colaboración incluida en el dossier del profesor Alfons Martinell - uno de nuestros más hondos conocedores de la evolución de las políticas locales para la cultura - formulada a modo de decálogo, nos ofrece una visión amplia de un conjunto de referentes históricos determinantes del proceso de estas décadas pasadas, el recordatorio del peso constante del esfuerzo municipal en el sostenimiento de los servicios culturales, las carencias legislativas y políticas que lastran las capacidades de los Ayuntamientos y los peligros globales que afectan a la cultura, sugiriendo una serie de vías para abordar este reto desde el principio de los derechos culturales. En el mismo sentido, preocupada por la falta de una estrategia de los mismos, la socióloga y activista Estefanía Rodero propone en su colaboración en este dossier, la necesidad de establecer un marco legal y real de derechos culturales como condición imprescindible para la acción cultural local, y nos recuerda también la importancia de las instituciones internacionales en el establecimiento de ese marco innovador, presupuestario y legislativo.
Los retos son extraordinarios. Reclaman respuestas estructurales, recargas conceptuales y adaptaciones a las nuevas formas de operar de la creación, el mercado y la ciudadanía. Unos procesos mediados de forma crucial por las mutaciones sobre procesos y miradas resultantes de la implantación de un nuevo universo de producción, intercambio, circulación y consumo. Cuando el escritor y crítico John Berger alentaba en su programa de televisión" "Modos de ver" o en sus textos $(2001,2016,2018)$ sobre los cambios en la percepción de la obra de arte tras la llegada de los medios de masas, no podía imaginar cuales serían las transformaciones de la mirada tras la universalización de la cultura digital, las redes sociales y sus aplicaciones más populares.

Ahora, tras la plena ocupación del mundo cultural por el mundo digital, se modifica de nuevo el papel del soporte y también del proceso mismo de lo cultural. Frente a las tesis de Walter Benjamin ${ }^{31}$ respecto a la unicidad de las obras culturales, la idea de la importancia del aquí y ahora que las hacía irrepetibles en el mundo de la creación digital —y, en palabras de la crítica cultural Mercedes Bunz (2007) — se produce una nueva mutación de consecuencias significativas: la lógica de la autenticidad ya no está reñida con la lógica de la repetición. La copia digital altera el orden establecido y da por rota la relación sobre la que se asentaba buena parte de la intermediación cultural hasta finales del pasado siglo, alterando la relación entre el original de la obra, su valor de unicidad que determina su identidad y aquello que la hace auténtica.

Este cambio, consustancial a la capacidad de penetración de la red en nuestras vidas cotidianas, no es sin embargo señal inexorable de que la cultura, en su variante digital, haya perdido capacidad transformadora y utópica. Bunz afirma y muestra cómo podemos observar vínculos evidentes entre el collage dadaísta o el espíritu irreverente del surrealismo con los actuales memes. También encontramos similitudes incontestables entre la sociedad del espectáculo descrita por Debord y los situacionistas y la actual sobreexposición de los pequeños hechos íntimos de nuestras vidas cotidianas en las redes sociales. O en la voluntad de proporcionar a cualquiera un altavoz desde el que romper el tejido multimedia en el que vive enredada la ciudadanía, que tanto trabajaron los culture-jammers $^{32}$, y que ya tenemos a nuestra disposición en forma de redes sociales, webs y demás herramientas digitales para crear, distribuir, cocrear o compartir. El mundo analógico y su lógica de la repetición han sido sustituidos por una realidad distinta, manipulable y cambiante, y la política cultural está obligada a transformarse y adaptarse a esa nueva situación sobrevenida. 


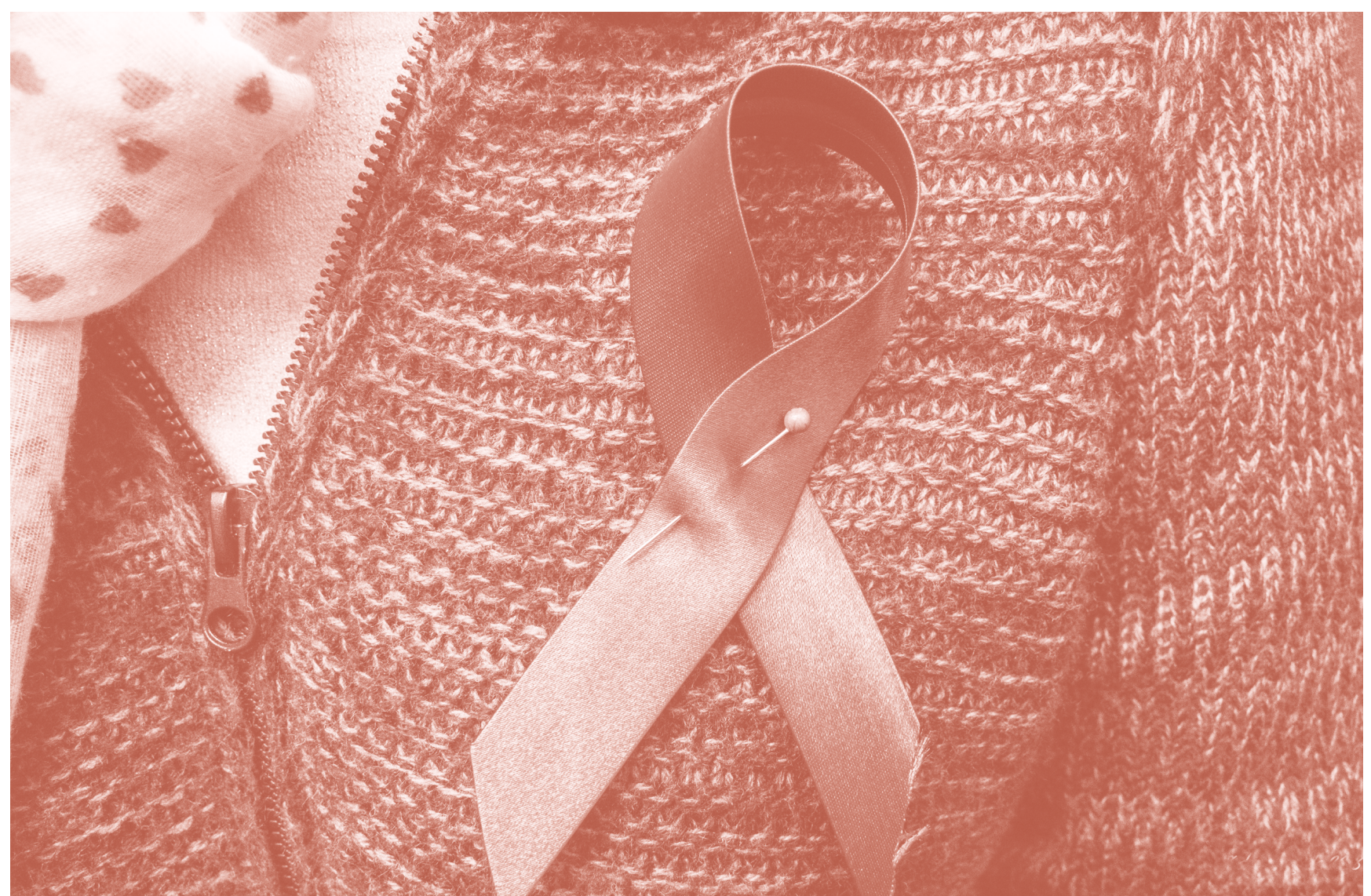

Planificar la ciudad sin la cultura como parte de su visión articuladora genera aún más graves desigualdades estructurales, que son muy difíciles de modificar más tarde. No habrá, como señalan las conclusiones del Encuentro de Valladolid, empoderamiento cultural sin el adecuado y preciso empoderamiento político; en la gestión cotidiana y en las redes. Pero, al tiempo, colocando la cultura en el lugar de su importancia, sin obsesiones hegemónicas programáticas ni fantasías de transversalidad exageradas. En palabras de Terry Eagleton (2017, pág. 178):

De hecho, los problemas centrales que afronta la humanidad al adentrarse en un nuevo milenio no son culturales en absoluto. Son mucho más mundanos y materiales que todo eso. Guerras, hambre, armas, genocidios, enfermedades, desastres ecológicos: todos tienen aspectos culturales, pero la cultura no es lo decisivo en ellos. Si quienes hablan de cultura no saben hacerlo sin hinchar el concepto, quizá sería mejor que permanecieran en silencio

Con todo, en medio de esa necesaria modestia, será necesario que instituciones como la Federación Española de Municipios y Provincias redoblen su esfuerzo a la hora de acompañar a los miles de municipios españoles en el camino de la recuperación de un papel para la cultura en el cambio local que, hasta hace dos décadas, cientos de ayuntamientos implicados venían cumpliendo. La Agenda 21 de la Cultura ${ }^{33}$, firmada y rubricada por muchos de ellos, no puede seguir siendo papel mojado. Juana Escudero, subdirectora de Educación y Cultura de la FEMP, nos describe en su aportación a este dossier, algunos de los caminos que, como profunda conocedora de la realidad local española, pueden ayudar a hacerlo posible.

Las transformaciones culturales hoy deben tener principio y esencia de género. En cada iniciativa cultural ha 
de estar presente una estrategia global que dé a las mujeres el espacio que en muchas ocasiones la tradición cultural ha impedido. Como señala en su trabajo para este número de Periférica Gemma Carbó, citando a Lori Saint Martin:

Era necesario también en cultura partir de una política de cuotas y presencia femenina en las programaciones pero para avanzar hacia una política de calidad que realmente considere la cuestión de género como eje transversal: desde las instituciones y a todos los niveles, será necesario contar con las mujeres para que se cuente con las mujeres [...] creo que queda del todo claro que es urgente situarnos en el paradigma de las políticas culturales desde un enfoque feminista y de derechos humanos, entendiendo que no podemos permitirnos el lujo de seguir hablando de democracia, sin que se respete, de hecho, la verdadera democracia cultural

Tecnológicas y artesanas al tiempo ${ }^{34}$, las formas del trabajo cultural han de transformar sus herramientas y objetivos. Entre tecnología y artesanía, la mediación cultural necesita volver a cumplir un papel transformador, ayudar a dotar de recursos propios a la ciudadanía que fortalezcan la conciencia de que solo la vida sostenible, igual, generosa, acogedora de lo distinto y capaces de contrarrestar la inmensa fuerza expulsora de las comunidades empeñadas en la civilización de la igual. Y para ello, las tareas de mediación han de abordar al tiempo trabajo práctico y teórico, puesto que, en palabras de Sennett (2013, pág. 13) «disociar el ámbito material del analítico tiene como consecuencia un debilitamiento intelectual, una disminución de nuestra capacidad de visión y de comprensión». Es poco discutible la falta de consolidación en España de los Estudios Culturales ${ }^{35}$ y su posterior conversión en formaciones profesionales despojadas de los valores de transformación y cambio propias de las políticas culturales de progreso, aunque este no sea asunto del presente artículo. Pero los cierto es que una parte importante del debilitamiento de la esfera cultural pública tiene este origen y reclama una respuesta.

En asuntos que afectan tan intensamente a la vida de la ciudadanía como los culturales, hoy definitivamente son factores imprescindibles los interfaces de acceso y de participación, que aceleran la generación de los mecanismos y condiciones para la expresión creativa, el contraste de opinión y las aportaciones de pluralidad inclusiva. En un mundo guiado por las oportunidades y condicionantes del espacio y de las herramientas digitales, será necesario un gran esfuerzo de imaginación y una ambiciosa estrategia de cambio para que la incorporación de la ciudadanía a esos territorios se produzca con las garantías de acceso y de participación, facilitadas hoy por la implantación casi total de las redes de comunicación y por los procesos y formas de integración de contenidos y experiencias. Y en ese objetivo las instituciones pueden ejercer un papel normativo y operativo esencial, poniendo a disposición y ayudando a la integración digital de las comunidades, especialmente las menos favorecidas, a través de los recursos de intermediación de los que dispone.

Esos interfaces son tanto digitales e intangibles como humanos y materiales: aplicaciones, programas, instalaciones, equipamientos y personas expertas, muchas de las cuales forman ya parte de las plantillas municipales en muchos ayuntamientos españoles. Y deben ser manejados por quienes conozcan a fondo sus utilidades, limitaciones y esclavitudes. Es decir, un enorme esfuerzo formativo en esta materia será necesario si deseamos contar con una intermediación cultural cualificada y competente en los próximos años. Y nuevas generaciones digitales, críticas y competentes tienen que incorporarse a un sector profesional cargado de experiencia pero también de conservadurismo. Si Evgeni Mozo-

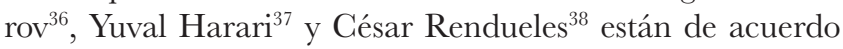
en algo, señalando las limitaciones, peligros y escenarios críticos que el mundo digital supone para la cultura democrática y de proximidad, habrá que pensar que puede que no se hayan equivocado.

En esta mutación de la intermediación, los nuevos usos de los equipamientos culturales, sobredimensionados o erróneamente diseñados muchos de ellos, incapaces de servir al interés general en su actual modo de gestión, reclaman también una atención específica. Tanto para la crítica de su funcionamiento como para la reconceptualización de sus usos, Juan Arturo Rubio, ya citado en páginas anteriores y uno de nuestros más comprometidos investigadores de políticas culturales comparadas, reflexiona en su aportación a este dossier en torno a la ascensión y caída de las arquitecturas culturales del poder local y el necesario futuro de instalaciones necesarias para la democracia cultural pero hoy convertidas en contenedores de escasos contenidos.

La subsistencia de las economías de lo pequeño, mutadas por el impacto digital, requerirá también que cualquier municipio, más o menos grande, sea capaz de cambiar sus formas de hacer hasta conseguir diseñar e implementar, colaborativamente, una estrategia integral para la consolidación de su sector cultural y creativo en todas sus fases, desde la expe- 
rimentación hasta la comercialización o los usos compartidos. Porque atender a las industrias culturales no es esencialmente tan solo una tarea cultural, sino que es, sobre todo, parte de una política económica local de aprovechamiento del valor endógeno de estas, como un elemento más de una economía sostenible. La experimentación, imprescindible para el desarrollo de una cultura participativa y abierta, capaz de romper con el tsunami del entretenimiento, ha de estar siempre presente en esos procesos para garantizar que el factor transformador no queda obliterado por el poder de la apisonadora del consumo.

Hemos de seguir entendiendo nuestros propios factores de identidad como punto de partida imprescindible para una vida cultural local decente: devolviendo espacio y tiempo a la historia y la cultura cercana, invisibles casi siempre y recuperando la dignidad y capacidad de representación del patrimonio inmaterial y material de la vida en común. Esto, añadido a nuestro tradicional patrimonio cultural, constituye un motivo de orgullo compartido: lenguas, tradiciones, prácticas comunitarias cuyas transformaciones han de procurar evitar la fácil tentación de la festivalización y la banalización, la invención falsaria, la manipulación política populista constante en estos tiempos o la pendiente de la espectacularización histórica, tan frecuentes como tramposas para la historia en tantos casos.

\section{Coda}

De estos y otros temas cruciales de la agenda cultural local hablan los artículos que siguen. Recordar tan solo que ahora, lo necesario será recuperar una conversación de múltiples orígenes, apasionada y crítica, capaz de hacer que, por fin, como casi ocurrió hace cuatro décadas, se encuentren tantas voces que hoy circulan por docenas de canales paralelos en el campo abierto de la esfera pública de la cultura y que apenas se reconocen entre sí.

\section{Notas}

(1) CUETO ALAS, J. (1982): Mitologías de la modernidad, Barcelona, Salvat, pág. 63.

(2) Surveillance capitalism, capitalismo de control y vigilancia, es un término que comienza a aparecer en los estudios culturales y las ciencias políticas en la década de los ochenta y que recientemente ha recuperado visibilidad gracias a la publicación de la obra The Age of Surveillance Capitalism de Shoshana Zuboff en la que se analiza el fenómeno de Google y las empresas de captación, catalogación y distribución de la información personal, el fenómeno del Big Data y los efec- tos de manipulación y control social inherentes. Otras clasificaciones del actual entorno productivo que ordena la vida de las comunidades, como la de capitalismo de plataformas (SRNICEK, 2018) nos proponen aproximaciones críticas similares al fenómeno.

(3) Una buena introducción al tema y a sus impactos en la cultura, también en la de proximidad, puede encontrarse en este artículo del profesor David Lyons (2018), colaborador de Zygmut Baumann y responsable de la Cátedra de Estudios de la Vigilancia en la Universidad de Queens, (Kingston, Canada) https://www.researchgate.net/publication/327423410_Surveillance_capitalism_surveillance_culture_and_data_politics

(4) Uno de los muchos conceptos que distintas instituciones y países han venido adoptando como forma de medida de la satisfacción ciudadana es el Índice de Felicidad. Desde 2012, Naciones Unidas publica un Índice Mundial de la Felicidad (http://wordhappiness.report) que en España tiene su réplica en el estudio de La Caixa: https://observatoriosociallacaixa.org/es/-/el-impacto-de-la-cultura-y-el-ocio-en-la-felicidad-de-los-espanoles [fecha de consulta: 07/08/2019]

(5) La Fundación Alternativas, en colaboración con la FEMP y el Ministerio de Cultura, destinó su volumen para 2019 del Informe sobre el estado de la Cultura en España a las políticas locales, bajo el título Cultura local, democracia, desarrollo. En él colaboran algunas de las firmas que participan también en este dossier. El documento íntegro puede consultarse en: https://www.fundacionalternativas.org/public/storage/publicaciones_archivos/36e8ddaea9599809a584485d2c4bbbe8.pdf

(6) RUBIO AROSTEGUI, J. y RÍUS ULLDEMOLINS, J. (2016): El diagnóstico de la crisis de la cultura en España: del recorte público a la crisis sistémica, Arte, Individuo y Sociedad, n 28 (1), págs. 41-57.

(7) Para una definición precisa de la esfera pública de la cultura ver MCGUIGAN, J. (2005): The Cultural Public Sphere, European Journal of Cultual Studies, $\mathrm{n}^{\circ} 8$ (4), págs. 427-443, https://journals.sagepub.com/ doi/10.1177/1367549405057827

(8) Un listado de algunas de sus publicaciones es accesible en la siguiente dirección: https://dialnet.unirioja.es/ servlet/autor? codigo $=3164551$

(9) Pueden verse muchos de sus trabajos en este repositorio: https://dialnet.unirioja.es/servlet/autor?codigo $=1203929$

(10) Algunos de sus trabajos pueden verse en: https:// dialnet.unirioja.es/servlet/autor?codigo $=1799364$ 
(11) Varias de las publicaciones de Rausell pueden consultarse en https://dialnet.unirioja.es/servlet/autor?codigo $=200608$

(12) Revista OIKOS: https://dialnet.unirioja.es/servlet $/$ revista? codigo $=8140$

(13) Revista Debats: https://dialnet.unirioja.es/servlet $/$ revista? codigo $=403$

(14) Arte, Individuo y Sociedad: https://dialnet.unirioja.es/servlet $/$ revista?codigo $=1524$

(15) VV. AA. (2012): CT o la Cultura de la Transición. Crítica a 35 años de cultura española. Barcelona, Random House Mondadori.

(16) VV. AA. (2002): La politica cultural en el Municipio. El respeto a los derechos de propiedad intelectual. Madrid, SGAE/ FEMP.

(17) VV. AA. (1977): Cultura para 70.000. Universidad Popular de Rekaldeberri, Madrid, Editorial Nuestra Cultura.

(18) En 1979 Joaquin Jordá filmó un documental sobre los trabajadores de la fábrica de electrodomésticos $\mathrm{Nu}$ max que desde 1977 hasta 1979, ocuparon y autogestionaron la fábrica como respuesta al intento de cierre irregular por parte de sus propietarios, filonazis refugiados en Barcelona en los años cincuenta.

(19) Para saber algo más de Eduard Delgado, véase: http://interaccio.diba.cat/wiki/delgado-clavera-eduard

(20) BUSTAMANTE, E. (ed). (2013): Industrias creativas: Amenazas para la cultura digital. Barcelona, Gedisa editorial.

(21) MARTINEZ ILLA, S. y RÍUS ULLDEMOLINS, J. (2012): La política cultural de la Diputación de Barcelona. La influencia del contexto político institucional y de la articulación entre administraciones en el desarrollo de un modelo singular de cooperación cultural, Revista RIPS, ISSN 1577-239X, volumen 11, nº 1, págs. 9-37, fecha de consulta: 26/09/19, http://www.usc. es/revistas/index.php/rips/article/view/857

(22) Podemos encontrar una descripción detallada del concepto «capital cultural» en: THROSBY, D. (2008): Economía y cultura, México DF, Consejo Nacional para la Cultura y las Artes, págs. 73-92.

(23) http://www.nesta.org.uk/publications/cultural-policy-time-creative-industries [fecha de consulta: 26/09/2019].

(24) El Informe Warwick sobre el futuro de la cultura plantea un panorama de situaciones críticas, prioridades y convivencias para la cultura británica que guarda cierta similitud con el debate pendiente en nuestro país respecto a la convivencia de las políticas de derechos culturales y las destinadas a la industria cultural y creativa. Las conclusio- nes y recomendaciones tratan de evitar un posicionamiento radical frente a la preminencia absoluta de esas industrias culturales y creativas en las políticas públicas del país, proponiendo vías para la recuperación de los derechos culturales de la comunidad y el desarrollo de la economía del sector. Puede consultarse el informe en: https://warwick.ac.uk/ research/warwickcommission/futureculture/finalreport/ warwick_commission_final_report.pdf

(25) En un poco sospechoso paper realizado por el grupo de expertos (OMC) de los estados miembros de la Unión Europea sobre la participación cultural en 2012, se señala en su introducción (pág. 5) que: «De hecho, el acceso a la cultura es un asunto de constante debate en Europa. Los datos disponibles de la participación cultural muestran como una parte significativa de la población, (con diferencias muy sustanciales según los países). No participa aún en actividades culturales populares como la asistencia al cine o la lectura de libros. Los porcentajes de participación tienden a ser mucho más bajos en actividades como la visita a museos, con ciudadanos en circunstancias socialmente de riesgo (en términos de educación y niveles de ingresos) participando mucho menos que aquellas personas con perfiles educativos más altos y mayores ingresos». Puede consultarse en la siguiente dirección: https://ec.europa.eu/assets/eac/culture/policy/ strategic-framework/documents/omc-report-access-to-culture_en.pdf

(26) FERNÁNDEZ LEÓN, J. (2018): “¿Para qué sirve la longitud del brazo? Una aproximación al origen de las políticas culturales en el mundo anglosajón". Periférica Internacional. Revista para el análisis de la cultura y el territorio, $\mathrm{n}^{\circ} 18$, https://revistas.uca.es/index.php/periferica/article/view/ 418ttp://dx.doi.org/10.25267/Periferica.2017.i18

(27) Una concisa descripción del proceso puede encontrarse en ROWAN, J, (2010): Emprendizajes en Cultura, Madrid, Traficantes de Sueños.

(28) Tal ha sido la capacidad de penetración de la idea que la propia UE ha definido su programa antes destinado a la Cultura como Europa Creativa: https://ec.europa.eu/ programmes/creative-europe/node_es

(29) Los documentos elaborados por el último Gobierno del Partido Popular en España son una buena muestra de limitación intelectual y política, a la vez catálogo de lugares comunes y buenas intenciones. Pueden consultarse, por ejemplo El Plan estratégico General de la Secretaría de Estado de Cultura (2012): https:// sede.educacion.gob.es/publiventa/plan-estrategico-general-2012-2015-secretaria-de-estado-de-cultura/cultu- 
ra-sociedad/14133C y el Plan Cultura 2020 (elaborado en 2017): https://www.culturaydeporte.gob.es/mcd/ dam/jcr:27 7e 1da9-67bf-4c41-8e03-a30fdb 1bd839/ plan-cultura-2020.pdf

(30) BBC (1972): Ways of Seeing. Los capítulos están disponibles con subtitulación, pudiendo visionarse el primero de ellos en: https://www.youtube.com/watch?reload=9\&$\mathrm{v}=2 \mathrm{~km} 4 \mathrm{IN} \_$udlE

(31) BENJAMIN, W. (2008): "La obra de arte en la época de su reproductibilidad técnica (primera redacción)" En Obras Libro I, n ${ }^{\circ}$ 2, Madrid, Abada, pág. 13.

(32) Wikipedia. (2019): Culture jamming, fecha de consulta: 07/10/19, https://en.wikipedia.org/wiki/Culture_ jamming

(33) Para acceder a documentos e iniciativas de la Agenda 21 que pueden servir de guía de trabajo puede consultarse: https://ec.europa.eu/programmes/creative-europe/node_es

(34) SENNETT, R. (2013): Artesanía, tecnología y nuevas formas de trabajo, Buenos Aires, Madrid, Katz/CCGB.

(35) Para el análisis de los problemas de la formación de formadores universitarios en España en esta disciplina y los problemas de los Estudios Culturales en España, ver: CORNUT-GENTILLE D'ARCY, C. (2013) Los Estudios Culturales en España. Exploraciones teórico-conceptuales desde el límite disciplinar. Valencia, Aduana Vieja.

(36) MOROZOV, E. (2019): "Internet: la utopía escondía negocio y vigilancia", El País, https://elpais.com/elpais/2019/05/03/ideas/1556900343_961366.html

(37) HARARI, Y. N. (2019): "Los cerebros 'hackeados' votan", El País, https://elpais.com/internacional/2019/01/04/actualidad/1546602935_606381.html

(38) RENDUELES, C. (2018): "Herejes de la religión digital", El País, https://elpais.com/cultura/2018/09/13/ babelia/1536828693_419316.html

\section{Bibliografía seleccionada}

BENJAMIN, W. (2008): Obras. Libro I, Madrid, Abada.

BUNZ, M. (2007): La utopía de la copia. El pop como irritación, Buenos Aires, Interzona.

BYUNG-CHUL, H. (2017): La expulsión de lo distinto, Barcelona, Herder.

COSTA, J. (2018): Cómo acabar con la contracultura. Una historia subterránea de España, Madrid, Taurus.

CRUZ, R. y PÉREZ LDESMA, M. (eds.) (1997): Cultura y movilización en la España contemporánea, Madrid, Alianza Editorial.
EAGLETON, T. (2017): Cultura, Madrid, Taurus.

FRANCO, J. (2004): Memorias del tío Fess, Madrid, Aguilar.

GARCÍA PELAYO, I. y J. (2003): La fabulosa historia de los Pelayo, Barcelona, Plaza y Janés.

GRACIA, J. (2006): Estado y cultura. El despertar de una conciencia crítica contra el franquismo, 1940-1962, Barcelona, Anagrama.

- (2009): Más es más. Sociedad y cultura en la España democrática (1986-2008). La casa de la riqueza, Madrid, Iberoamericana Editorial Vervuert.

GUNTÍN, F. (1998): L`art contemporani des de l'àmbit local, Barcelona, Pages/Diputació de Barcelona.

HEWISON, R. (1987): The Heritage Industry, London, Methuen.

HORNE, D. (1984): The Great Museum, London, Pluto Press.

(1986): The Public Culture, The thriumph of industrialism, London, Pluto Press.

LÓPEZ DE AGUILERA, I. (2000): Cultura y ciudad. Manual de política cultural municipal, Gijón, Ediciones Trea.

MALVIDO, P. (2004): Nosotros los malditos., Barcelona, Anagrama.

MARZO, J. y MAYAYO, P. (2015): Arte en España (19392015) ideas, prácticas, políticas, Madrid, Ediciones Cátedra.

MCGUIGAN, J. (2004): Rethinking cultural policy, Maidenhead, Open University.

MENGER, P. (2010): Cultural policies in europe. from a state to a city-centered perspective on cultural generativity, Tokyo, National Graduate Institute for Policy Studies.

MOROZOV, E. (2013): To save everything, click here, New York, PublicAffairs.

MURGA CASTRO, I. y LÓPEZ SÁNCHEZ, J. (eds.) (2016): Política cultural de la Segunda República Española, Madrid, Editorial Pablo Iglesias.

NAZARIO (2016): La vida cotidiana del dibujante underground, Barcelona, Anagrama.

lona, Anagrama. (2018): Sevilla y la casa de las pirañas, Barce-

ORDOVÁS, J. (2017): Fiebre de vivir. Apocalípticos y desintegrados en el rock español de los 70, Valencia, Efe eme.

RAMÍREZ, J. A. (ed) (2010): El sistema del arte en España, Madrid, Cátedra.

RAUSELL KÖSTER, P. (dir.) (2007): Cultura. Estrategia para el desarrollo local, Madrid, MAEG/AECI.

RENDUELES, C. (2013): Sociofobia: El cambio politico en la era de la utopia digital, Madrid, Capitán Swing. 
RIUS ULLDEMOLÍNS, J. y GISBERT, V. (2018): "¿Por qué las políticas culturales locales no cambian? Constricciones del modelo urbano, inercia en la gestión y batallas culturales en los «gobiernos del cambio» en Madrid y Barcelona (2015-2018)", Revista Española de Ciencia Política, n 47 , págs. 93-122, https://doi.org/10.21308/recp.47.04

ROWAN, J. (2010): Emprendizajes en cultura, Madrid, Traficantes de sueños.

RUBIO ARÓSTEGUI, J. (2003): La política cultural del Estado en los gobiernos socialistas: 1982-1996, Gijón, Ediciones Trea. RUBIO ARÓSTEGUI, J. y RÍUS ULLDEMOLÍNS, J. (2016): Treinta años de políticas culturales en España, Valencia, Universitat de Valencia.
(2016): El diagnóstico de la crisis de la cultura en España: del recorte público a la crisis sistémica, Revista Arte, Individuo y Sociedad, 28(1) 41-57

SRNICEK, N. (2016): Platform Capitalism, London, Polity Press.

VOGEL, A. (2017): Bikinis, futbol y rock \& Roll. Crónica pop bajo el franquismo sociológico, Madrid, Foca.

VV. AA. (1982): Curtó-1. Cultura i municipi, Barcelona, CopyArt.

(2015): El futuro de los centros culturales en la Europa Creativa, Camargo, Ayuntamiento de Camargo.

ZAFRA, R. (2017): El entusiasmo. Precariedad y trabajo creativo en la era digital, Barcelona, Anagrama. 